\title{
Optimal representation of non-stationary random fields with finite numbers of samples: A linear MMSE framework
}

\author{
Ayça Özçelikkale*, Haldun M. Ozaktas \\ Department of Electrical Engineering, Bilkent University, TR-06800, Ankara, Turkey
}

\section{A R T I C L E I N F O}

\section{Article history:}

Available online 7 May 2013

\section{Keywords:}

Random field estimation

Non-stationary signals

Uniform sampling

Gaussian-Schell model

\begin{abstract}
A B S T R A C T
In this article we consider the representation of a finite-energy non-stationary random field with a finite number of samples. We pose the problem as an optimal sampling problem where we seek the optimal sampling interval under the mean-square error criterion, for a given number of samples. We investigate the optimum sampling rates and the resulting trade-offs between the number of samples and the representation error. In our numerical experiments, we consider a parametric non-stationary field model, the Gaussian-Schell model, and present sampling schemes for varying noise levels and for sources with varying numbers of degrees of freedom. We discuss the dependence of the optimum sampling interval on the problem parameters. We also study the sensitivity of the error to the chosen sampling interval.
\end{abstract}

(c) 2013 Elsevier Inc. All rights reserved.

\section{Introduction}

In this article we investigate certain trade-offs in the representation of random fields. We consider the representation of a finite-energy non-stationary random field with a finite number of samples. We study the optimum sampling rates and the trade-offs between the number of samples and the representation error.

We may summarize our general framework as follows: We consider equidistant sampling of non-stationary signals with finite energy. We are allowed to take only a finite number of samples. We investigate the optimal representation of the field with these finite number of samples under the mean-square error criterion. We seek the optimal sampling interval for a given number of samples. We deal with questions such as "At least how many samples should we take to achieve a given level of error?", "What is the minimum error that can be achieved with a given number of samples?", and "How sensitive is the error to the sampling interval?". We are not able to offer complete analytical solutions to these problems, but we design sampling schemes that provide insight into the answers of such questions.

An important aspect of our formulation is the restriction of the total number of samples to be finite. Although several aspects of the sampling of random fields are well understood (mostly for stationary fields and also for non-stationary fields), most studies deal with the case where the number of samples per unit time is finite (and the total number of samples are infinite).

\footnotetext{
* Corresponding author. Fax: +90 3122664192.

E-mail addresses: ayca@ee.bilkent.edu.tr (A. Özçelikkale), haldun@ee.bilkent.edu.tr (H.M. Ozaktas).
}

We now review a number of representative works related to sampling of a random signal. A fundamental result in this area states that the Shannon-Nyquist sampling theorem, which is generally given for deterministic signals, can be generalized to widesense stationary (WSS) signals: A band-limited WSS signal can be reconstructed in the mean-square sense from its equally-spaced samples taken at the Nyquist rate [1]. In [2] a generalization of this result is provided for multiband signals. Generalizations of this result where the samples differ from ordinary Nyquist samples have also been considered: [3,4] offer various conditions under which error-free recovery is possible. $[5,6]$ show how much each sample point may be shifted before error-free recovery is no longer possible. A formal treatment of this subject in a general framework may be found in [6]. Methods for spectral analysis of nonuniformly sampled data are reviewed in [7]. In [8], the truncation error associated with the sampling expansion is studied. An average sampling theorem for band-limited random signals is presented in [9]. [10] further generalizes the Shannon-Nyquist sampling theorem to non-stationary random fields; [11] clarifies the conditions in [10]. $[12,13]$ consider sampling of varying classes of non-stationary signals using Loève bifrequency spectrum. [14] reviews results related to cyclostationarity in both deterministic and random frameworks. Recovery of random signals from observations of a noisy or filtered version of the unknown signal is also considered; such as [15] which focuses on interpolation of the input signal from samples of the output, and [16] which focuses on a noisy measurement scenario with a total bit constraint.

Another important aspect of our framework is the non-stationary signal model. A broad class of physical signals may be better represented with non-stationary models rather than stationary models, which has resulted in increasing interest in these 
models [17]. Although some aspects of the sampling of nonstationary fields are understood, such as the sampling theorem of [10], our understanding of non-stationary fields falls short of our understanding of stationary fields. Our purpose is to contribute to a better understanding of the trade-offs in the representation of non-stationary random fields.

In Section 2, we present the mathematical model of the problem considered in this article. The signal model we use in our experiments, Gaussian-Schell model, is discussed in Section 3. In Section 4 we present the numerical experiments. We conclude in Section 5 .

\section{Problem formulation}

In the specific measurement scenario under consideration in this paper, a signal corrupted by noise is sampled to provide a representation of the signal with finite number of samples. More precisely, the sampled signal is of the form

$g(x)=f(x)+n(x)$,

where $x \in \mathbb{R}, f: \mathbb{R} \rightarrow \mathbb{C}$ is the unknown proper Gaussian random field (random process), $n: \mathbb{R} \rightarrow \mathbb{C}$ is the proper Gaussian random field denoting the inherent noise, and $g: \mathbb{R} \rightarrow \mathbb{C}$ is the proper Gaussian random field to be sampled in order to estimate $f(x)$. We assume that $f(x)$ and $n(x)$ are statistically independent zero-mean random fields. We consider all signals and estimators in the bounded region $-\infty<x_{L} \leqslant x \leqslant x_{H}<\infty$. Let $D=\left[x_{L}, x_{H}\right]$ and $D^{2}=\left[x_{L}, x_{H}\right] \times\left[x_{L}, x_{H}\right]$. Let $K_{f}\left(x_{1}, x_{2}\right)=E\left[f\left(x_{1}\right) f^{*}\left(x_{2}\right)\right]$, and $K_{n}\left(x_{1}, x_{2}\right)=E\left[n\left(x_{1}\right) n^{*}\left(x_{2}\right)\right]$ denote the covariance functions of $f(x)$ and $n(x)$, respectively. Here ${ }^{*}$ denotes complex conjugation. We assume that $f(x)$ is a finite-energy random field, $\int_{-\infty}^{\infty} K_{f}(x, x) d x<\infty$, and $K_{n}(x, x), x \in D$ is bounded.

$M$ samples of $g(x)$ are taken equidistantly with the sampling interval $\Delta$ at $x=\xi_{1}, \ldots, \xi_{M} \in \mathbb{R}$, with $\Delta=\xi_{i+1}-\xi_{i}$, $i=1, \ldots, M-1$. Hence we have $g_{i} \in \mathbb{C}$ observed according to the model $g_{i}=g\left(\xi_{i}\right)$, for $i=1, \ldots, M$. By putting the sampled values in vector form, we obtain $\mathbf{g}=\left[g\left(\boldsymbol{\xi}_{1}\right), \ldots, g\left(\boldsymbol{\xi}_{M}\right)\right]^{\mathrm{T}}$. Let $\mathbf{K}_{\mathbf{g}}=E\left[\mathbf{g g}^{\dagger}\right]$ be the covariance matrix of $\mathbf{g}$, $\dagger$ denotes the conjugate transpose.

We note that contrary to standard textbook formulations of sampling, here the unknown signal is not modeled as a deterministic function. Rather, we consider a stochastic framework, and would like to estimate the signal $f(x), x \in D$, which is interpreted as a realization of a random field. Hence for each $x, f(x)$ is a random variable whose probability distribution function depends on $x$. Thus, for a given $\xi$, each sample $g(\xi)$ is also a random variable. Therefore, for a given set of sampling locations $\xi_{1}, \ldots, \xi_{M}$, the problem of recovering $f(x), x \in D$ is an estimation problem, where given a vector of random variables $\mathbf{g}=\left[g\left(\xi_{1}\right), \ldots, g\left(\xi_{M}\right)\right]^{\mathrm{T}}$, we would like to estimate $f(x)$, for each $x \in D$. An introduction to these types of stochastic estimation formulations can be found in [18, Ch. 5].

The vector $\mathbf{g}$ provides a representation of the random field $f(x)$. We do not have access to the true field $f(x)$ but we can find $\hat{f}(x \mid \mathbf{g})$, the minimum mean-square error (MMSE) estimate of $f(x)$ given $\mathbf{g}$. For a given maximum allowed number of sampling points $M_{b}$, our objective is to choose the location of the samples $\left(\xi_{1}, \ldots, \xi_{M} \in \mathbb{R}, M \leqslant M_{b}\right)$, so that the MMSE between $f(x)$ and $\hat{f}(x \mid \mathbf{g})$ is minimum. This problem can be stated as one of determining

$\boldsymbol{\varepsilon}\left(M_{b}\right)=\min _{\Delta, x_{0}} E\left[\int_{D}|f(x)-\hat{f}(x \mid \mathbf{g})|^{2} d x\right]$,

subject to $M \leqslant M_{b}$. Here the samples are taken around the midpoint $x_{0}=0.5\left(\xi_{1}+\xi_{M}\right)$, which along with $\Delta$ we allow to be op- timally chosen. We now discuss certain features of Eq. (2). Further discussion will be given in Section 4 .

Noting that the observed values are in vector form, the linear estimator for (2) can be written as [18, Ch. 6]

$\begin{aligned} \hat{f}(x \mid \mathbf{g}) & =\sum_{j=1}^{M} h_{j}(x) g_{j} \\ & =\mathbf{h}(x) \mathbf{g}\end{aligned}$

where the function $\mathbf{h}(x)=\left[h_{1}(x), \ldots, h_{M}(x)\right]$ satisfies the equation

$\mathbf{K}_{f \mathbf{g}}(x)=\mathbf{h}(x) \mathbf{K}_{\mathbf{g}}$,

where $\mathbf{K}_{f \mathbf{g}}(x)=E\left[f(x) g^{\dagger}\right]=\left[E\left[f(x) g_{1}^{*}\right], \ldots, E\left[f(x) g_{M}^{*}\right]\right]$ is the cross covariance between the input field $f(x)$ and the measurement vector g. To determine the optimal linear estimator, one should solve (5) for $\mathbf{h}(x)$.

The error can be written more explicitly as

$$
\begin{aligned}
& E\left[\int_{D}|f(x)-\hat{f}(x \mid \mathbf{g})|^{2} d x\right] \\
& \left.=\left.E\left[\int_{D} \mid f(x)-\mathbf{h}(x) \mathbf{g}\right)\right|^{2} d x\right] \\
& \left.=\left.\int_{D} E[\mid f(x)-\mathbf{h}(x) \mathbf{g})\right|^{2}\right] d x \\
& =\int_{D}\left(K_{f}(x, x)-2 \mathbf{K}_{f \mathbf{g}}(x) \mathbf{h}(x)^{\dagger}+\mathbf{h}(x) \mathbf{K}_{\mathbf{g}} \mathbf{h}(x)^{\dagger}\right) d x \\
& =\int_{D}\left(K_{f}(x, x)-\mathbf{K}_{f \mathbf{g}}(x) \mathbf{h}(x)^{\dagger}\right) d x .
\end{aligned}
$$

Before leaving this section, we would like to discuss a few aspects of our formulation. We note that we do not assume that the fields are stationary and our formulation covers the general case including non-stationary fields. The covariance function of a stationary field depends only on the distance between two points. Here, however, such assumptions regarding the covariance function are not made; hence our formulation covers the general case including non-stationary fields. In sampling problems, a common approach is to assume that the field is stationary and effectively bandlimited, and use approaches based on the classical Nyquist sampling theorem. In that approach, interpolation formulas involving sinc functions are traditionally used; that is, the interpolating functions in Eq. (3) are assumed to be sinc functions, and the whole formulation is based on this assumption. Here we do not restrict ourselves to this case, and the estimators can be more general.

We note that the assumption of Gaussian fields makes the MMSE estimator and the error analytically tractable, which could be nonlinear and difficult to obtain for an arbitrary probability distribution. Nevertheless, even for an arbitrary distribution, the best linear mean-square error estimator is the same as the MMSE estimator for a Gaussian field when the mean and covariance functions are the same. (The estimator is called linear if $f(x \mid \mathbf{g})$ is a linear function of $\mathbf{g}$, and the best linear estimator is the one that minimizes the mean-square error over all such estimators.) Hence our formulation covers the case of non-Gaussian random fields as well. We also note that classical truncated sinc interpolation, being linear, is a special case of our formulation.

We now comment on the contribution to the error introduced by estimating the signal only in a bounded region. For notational convenience let $\hat{f}(x \mid \mathbf{g})$ be denoted as $\hat{f}(x)$. Let us define $\hat{f}_{D}(x)$ as 
$\hat{f}_{D}(x)=\hat{f}(x)$ for $x \in D$ and $\hat{f}_{D}(x)=0$ for $x \notin D$. Then, the error of representing $f(x)$ with $\hat{f}_{D}(x)$ can be expressed as

$$
\begin{aligned}
& E\left[\int_{-\infty}^{\infty}\left|f(x)-\hat{f}_{D}(x)\right|^{2} d x\right] \\
& =E\left[\int_{x \notin D}\left|f(x)-\hat{f}_{D}(x)\right|^{2} d x+\int_{x \in D}\left|f(x)-\hat{f}_{D}(x)\right|^{2} d x\right] \\
& =E\left[\int_{x \notin D}|f(x)|^{2} d x+\int_{x \in D}\left|f(x)-\hat{f}_{D}(x)\right|^{2} d x\right] \\
& =E\left[\int_{x \notin D}|f(x)|^{2} d x\right]+E\left[\int_{x \in D}\left|f(x)-\hat{f}_{D}(x)\right|^{2} d x\right] \\
& =\int_{x \notin D} K_{f}(x, x) d x+\boldsymbol{\varepsilon}\left(M_{b}\right) .
\end{aligned}
$$

Hence (13) states the following fact: The total error in representing the signal with $\hat{f}(x)$ in $x \in D$ without trying to estimate signal outside the region $D$, can be expressed as the sum of two terms: (1) a term denoting the total energy of the signal outside the interval $D$, (2) a term denoting the estimation error for the signal in the interval $D$. Since we have $\int_{-\infty}^{\infty} K_{f}(x, x) d x<\infty$, the first term can be made sufficiently small by taking a bounded but large enough region $D$ so that $\boldsymbol{\varepsilon}\left(M_{b}\right)$ becomes a good measure of representation performance over the entire line.

\section{Gaussian-Schell model}

Although the independent variable of the unknown signal in our problem may be time or some other variable, in this paper we choose our examples from optics, where the independent variable is often space. In our experiments we use a parametric non-stationary signal model known as the Gaussian-Schell model (GSM). This model is widely used in the study of random optical fields [19-21]. GSM beams have been investigated with emphasis on different aspects such as their coherent mode decomposition $[22,19]$, or their imaging and propagation properties [21,23-26]. Our results will shed some light on sampling trade-offs in the representation of these fields.

A Schell model source is characterized by the covariance function

$K_{f}\left(x_{1}, x_{2}\right)=I\left(x_{1}\right)^{0.5} I\left(x_{2}\right)^{0.5} v\left(x_{1}-x_{2}\right)$,

where $I(x)$ is called the intensity function and $v\left(x_{1}-x_{2}\right)$ is called the complex degree of spatial coherence in the optics literature. For a Gaussian-Schell model, both of these functions are Gaussian shaped

$I(x)=A_{f} \exp \left(-\frac{x^{2}}{2 \sigma_{I}^{2}}\right)$
$v\left(x_{1}-x_{2}\right)=\exp \left(-\frac{\left(x_{1}-x_{2}\right)^{2}}{2 \sigma_{v}^{2}}\right)$

where $A_{f}>0$ is an amplitude coefficient and $\sigma_{I}>0$ and $\sigma_{v}>0$ determine the width of the intensity profile and the width of the complex degree of spatial coherence, respectively. Hence the covariance function of a Gaussian-Schell model source takes the form

$K_{f}\left(x_{1}, x_{2}\right)=A_{f} \exp \left(-\frac{x_{1}^{2}+x_{2}^{2}}{4 \sigma_{I}^{2}}\right) \exp \left(-\frac{\left(x_{1}-x_{2}\right)^{2}}{2 \sigma_{v}^{2}}\right)$.

We note that as a result of the Gaussian shaped intensity profile; as we move away from the $x=0$, the variances of the random variables decay according to a Gaussian function. We also note that $v\left(x_{1}-x_{2}\right)$ is simply the correlation coefficient function which may be defined as $v\left(x_{1}-x_{2}\right)=\rho_{f}\left(x_{1}-x_{2}\right)=\frac{K_{f}\left(x_{1}, x_{2}\right)}{K_{f}\left(x_{1}, x_{1}\right)^{0.5} K_{f}\left(x_{2}, x_{2}\right)^{0.5}}$. Hence, as a result of the Gaussian shaped complex degree of spatial coherence function, the correlation coefficient between two points decays according to a Gaussian function as the distance between these two points increases.

$K_{f}\left(x_{1}, x_{2}\right)$ may be represented in the form

$K_{f}\left(x_{1}, x_{2}\right)=\sum_{k=0}^{\infty} \lambda_{k} \phi_{k}\left(x_{1}\right) \phi_{k}^{*}\left(x_{2}\right)$

where $\lambda_{k}$ are the eigenvalues and $\phi_{k}(x)$ are the orthonormal eigenfunctions of the integral equation $\int K_{f}\left(x_{1}, x_{2}\right) \phi_{k}\left(x_{1}\right) d x_{1}=\lambda_{k} \phi_{k}\left(x_{2}\right)$ $[22,19]$. Here we assume that the eigenvalues are indexed in decreasing order as $\lambda_{0} \geqslant \lambda_{1}, \ldots, \lambda_{k} \geqslant \lambda_{k+1}, \ldots, k \in Z_{+}$. In signal processing, this representation is known as the Karhunen-Loève expansion [27]. In statistical optics it is referred to as the coherent mode decomposition, where every eigenfunction is considered to correspond to one fully coherent (fully correlated) mode.

The eigenfunctions $\phi_{k}(x)$ for GSM sources are Hermite polynomials, whose exact form may be found in [19]. Since the eigenvalue distribution will play a crucial role in our investigations we will discuss them in detail. The ratio of the eigenvalue $\lambda_{n}$ to the lowest eigenvalue $\lambda_{0}$ is given by [19]

$\frac{\lambda_{n}}{\lambda_{0}}=\left(\frac{1}{\beta^{2}+1+\beta\left[(\beta / 2)^{2}+1\right]^{0.5}}\right)^{n}$

where $\beta$ is defined as

$\beta=\frac{\sigma_{v}}{\sigma_{I}}$

Here $\beta$ may be considered as a measure of the number of significant eigenvalues, hence the effective number of degrees of freedom (DOF) of the source. The effective DOF of a family of signals may be defined as the effective number of uncorrelated random variables needed to characterize a random signal from that family. The concept of the number of degrees of freedom is central to several works, such as [28-32]. It is known that the random variables that provide the best characterization of the source under the mean-square error criterion are the random variables with variances given by the eigenvalues associated with the KarhunenLoève expansion. Hence the spread of eigenvalues can be used to define the DOF of the signals. One can say that the DOF is lower when the eigenvalue distribution is more concentrated, and that the DOF is higher when the eigenvalue distribution is more uniformly spread. This definition may be made more precise by defining the effective DOF $D(\delta)$ as the smallest number satisfying $\sum_{i=1}^{D} \lambda_{i} \geqslant \delta \boldsymbol{\varepsilon}_{0}$, where $\delta \in(0,1]$ and $\boldsymbol{\varepsilon}_{0}=\int_{-\infty}^{\infty} K_{f}(x, x) d x=$ $\sum_{k \geqslant 0} \lambda_{k}<\infty$.

Returning to the Gaussian-Schell model, we note that as $\beta$ increases, the eigenvalues decay faster according to (19), so that the number of modes required to effectively represent the source decreases. Similarly, as $\beta$ decreases, the eigenvalues decay slower, and the number of modes required to effectively represent the source increases.

Before leaving this section, we would like to make a few remarks about the existence of the expansion in (18) for the GSM source. We note that, in general, sources defined on the infinite line do not have expansions with discrete eigenvalue spectrum. To obtain such an expansion, one usually considers the source on a compact region (which in our case corresponds to a bounded region). Then the existence of such a representation is guaranteed by Mercer's Theorem, see for example [33, Ch. 7]. In [19], an expansion with discrete eigenvalue spectrum is investigated for 


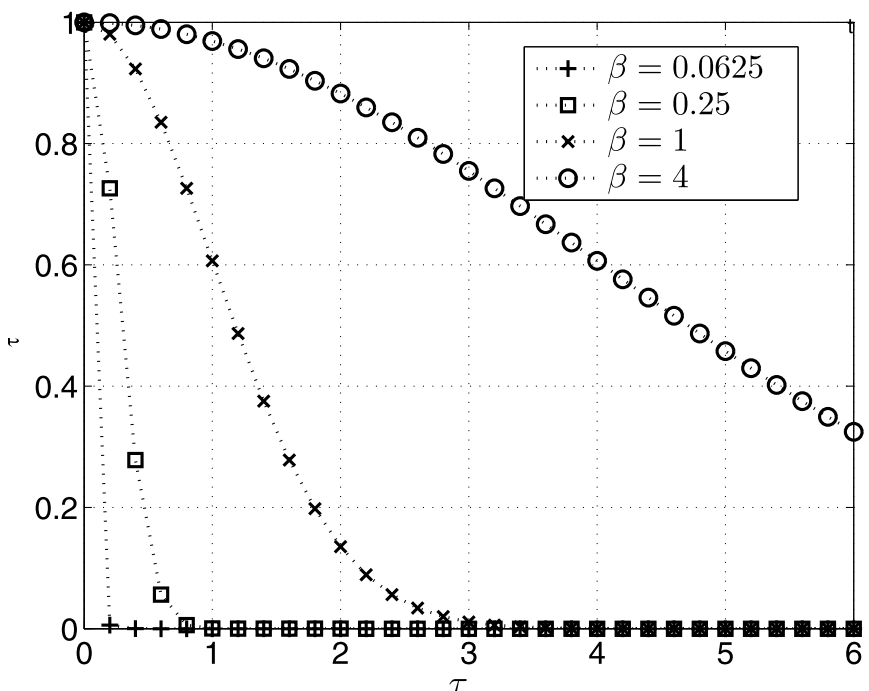

Fig. 1. Correlation coefficient as a function of distance for different values of $\beta$.

the GSM source on the infinite line without discussing the existence of such a decomposition in detail. Nevertheless, we here note that such an expansion is possible for the GSM source due to [34, Thm. 1]. This result states that along with continuity, having $\int_{-\infty}^{\infty} K_{f}(x, x) d x<\infty$ and $K_{f}(x, x) \rightarrow 0$ as $|x| \rightarrow \infty$ is sufficient to ensure such a representation. We note that both of these conditions are plausible in a physical context: the first one is equivalent to the finite-energy assumption and the second one requires the intensity of the field to vanish as $|x|$ increases, properties one commonly expects from physically realizable fields. As can be seen from (17), the covariance function of a GSM source satisfies these properties. Hence an expansion with a discrete eigenvalue spectrum as in (18) is possible for GSM sources.

\section{Experiments}

We now investigate the trade-off between the error and the number of samples, and the optimum sampling intervals associated with different sampling scenarios.

In our experiments, we choose to work with the equivalent parameters $\sigma_{I}$ and $\beta$, instead of $\sigma_{I}$ and $\sigma_{v}$. Under fixed $\beta$, this choice has the advantage of allowing the results for a given $\sigma_{I}$ value to be found by using the results for another $\sigma_{I}$ value, by appropriately scaling the coordinate space. Hence in our experiments we fix $\sigma_{I}=1$ without loss of generality.

To obtain covariance functions corresponding to random fields with varying DOF, we use different $\beta$ values: $\beta=1 / 16,1 / 4,1,4$. As stated in Section 3, $\sigma_{v}=\beta \sigma_{I}$ determines the width of the correlation function, which is a Gaussian function. We present the correlation function $\rho(\tau)$ for these values of $\beta$ in Fig. 1 .

We choose the noise model similar to the signal model, but with a flat intensity distribution: $I_{n}(x)=A_{n}, v_{n}\left(x_{1}-x_{2}\right)=$ $\exp \left(-\frac{\left(x_{1}-x_{2}\right)^{2}}{2 \sigma_{v, n}^{2}}\right)$, where $\sigma_{v, n}=\beta_{n} \sigma_{I}, \beta_{n}=1 / 32$. We consider different noise levels parameterized according to the signal-to-noise ratio, defined as the ratio of the peak signal and noise levels: $\mathrm{SNR}_{p}=\frac{A_{f}}{A_{n}}$. We consider the values $\mathrm{SNR}_{p}=0.1,1,10, \infty$ to cover a wide range of situations.

For simplicity in presentation, in our simulations we focus on $\Delta$ and set the less interesting $x_{0}=0$. We choose the interval $D$ equal to $\left[x_{L}, x_{H}\right]=\left[-5 \sigma_{I},+5 \sigma_{I}\right]$. With this choice of $D$, most of the energy of the signal falls inside the interval and the error arising from the fact that only signal values in the region $D$ are estimated is very small $\left(\leqslant 10^{-10}\right)$, so that the first term in (13)

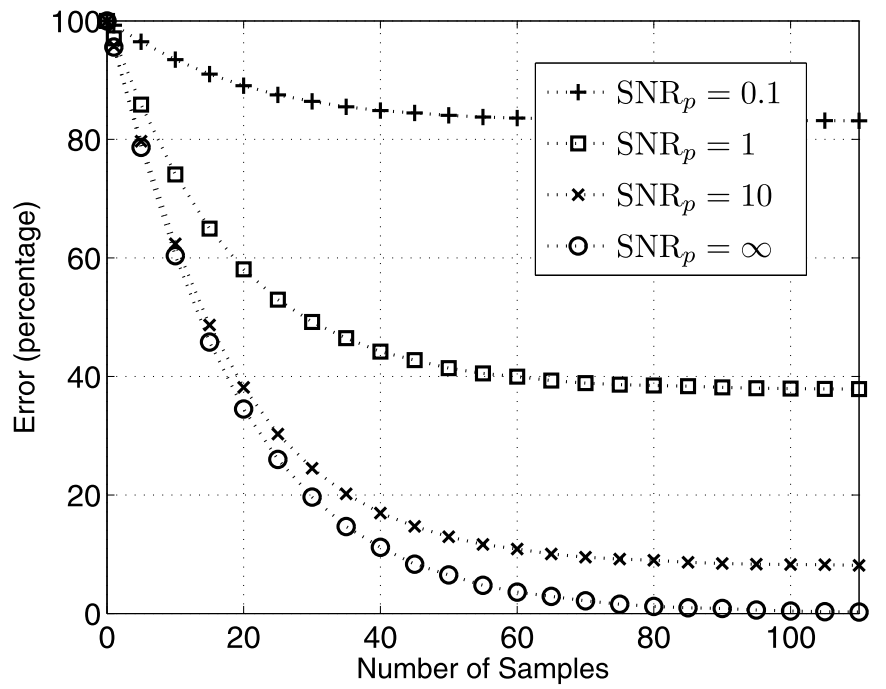

Fig. 2. Error vs number of samples, $\beta=0.0625$ (varying $\mathrm{SNR}_{p}$ ).

can be ignored. We discretize the $x$ space to compute the error expressions involving integrals over $x$. To solve (5) for $\mathbf{h}(x)$, we discretize (5) and approximate the solutions $h_{i}(x)$ as $\bar{h}_{i}(x)=$ $\sum_{j=1}^{N} h_{j i} \operatorname{sinc}\left(x-\mu_{j}\right)$ where $h_{j i}=h_{i}\left(x=\mu_{j}\right)$. Substitution of the approximate solution $\overline{\mathbf{h}}(x)=\left[\bar{h}_{1}(x), \ldots, \bar{h}_{M}(x)\right]$ into the right-hand side of (5) gives an expression that, in general, is not exactly equal to the left-hand side. We determine the parameters $h_{j i}$ by requiring (5) to hold exactly at $N$ selected points $v_{i}$. Hence (5) becomes a system of equations with $N \times M$ unknowns, $\mathbf{K}_{\overline{\mathbf{f g}}}=\mathbf{H} \mathbf{K}_{\mathbf{g}}$, where $H(i, j)=h_{i j}, \mathbf{K}_{\overline{\mathbf{f g}}}=E\left\{\overline{\mathbf{f}}^{\mathrm{T}}\right\}$, and $\overline{\mathbf{f}}=\left[f\left(v_{1}\right), \ldots, f\left(v_{M}\right)\right]$.

To find the optimum sampling intervals, we use a brute force method, where for a given $M_{b}$ we calculate the error for varying $\Delta$, and choose the one providing the best error value. This simple approach has the advantage of enabling us to investigate the effect of $\Delta$ on the error, and hence the sensitivity of the performance to choosing $\Delta$ different from the optimal values.

We report the error as a percentage defined as $100 \boldsymbol{\varepsilon}\left(M_{b}\right) / \boldsymbol{\varepsilon}_{0}$ where $\boldsymbol{\varepsilon}_{0}=\int_{-\infty}^{\infty} K_{f}(x, x) d x=A_{f} \sqrt{2 \pi}$.

\subsection{Trade-offs between the error and the number of samples}

In the following experiments we will investigate the trade-off between the MSE error $\boldsymbol{\varepsilon}\left(M_{b}\right)$ and $M_{b}$, the number of measurements we are allowed to make.

\subsubsection{Variable noise level}

We first investigate the effect of noise level on the trade-off between $\boldsymbol{\varepsilon}\left(M_{b}\right)$ and $M_{b}$. Here $\mathrm{SNR}_{p}$ takes the values $\mathrm{SNR}_{p}=$ $[0.1,1,10, \infty]$ and two different values of $\beta=[1 / 16,1]$ are considered. Figs. 2 and 3 correspond to $\beta=1 / 16$ (high effective DOF) and $\beta=1$ (low effective DOF), respectively. As expected, the error decreases with $M_{b}$ for both cases. We note that for both of cases, $\boldsymbol{\varepsilon}\left(M_{b}\right)$ is very sensitive to increases in $M_{b}$ for smaller $M_{b}$. Then it becomes less responsive and eventually saturates. For each value of $M_{b}$, the error decreases as $\mathrm{SNR}_{p}$ increases, and for higher $M_{b}$ values approaches zero as $\mathrm{SNR}_{p} \rightarrow \infty$. We see that when the field has low effective DOF (Fig. 3), we obtain much better trade-off curves for all values of $\mathrm{SNR}_{p}$ than Fig. 2, which represents the relatively high effective DOF case. For instance for $\mathrm{SNR}_{p}=\infty$, for the high DOF case an error of $20 \%$ is obtained when the number of samples is around 30, whereas for the field with low DOF a smaller error value is achieved with only 5 samples. This point is further investigated in the next section. 


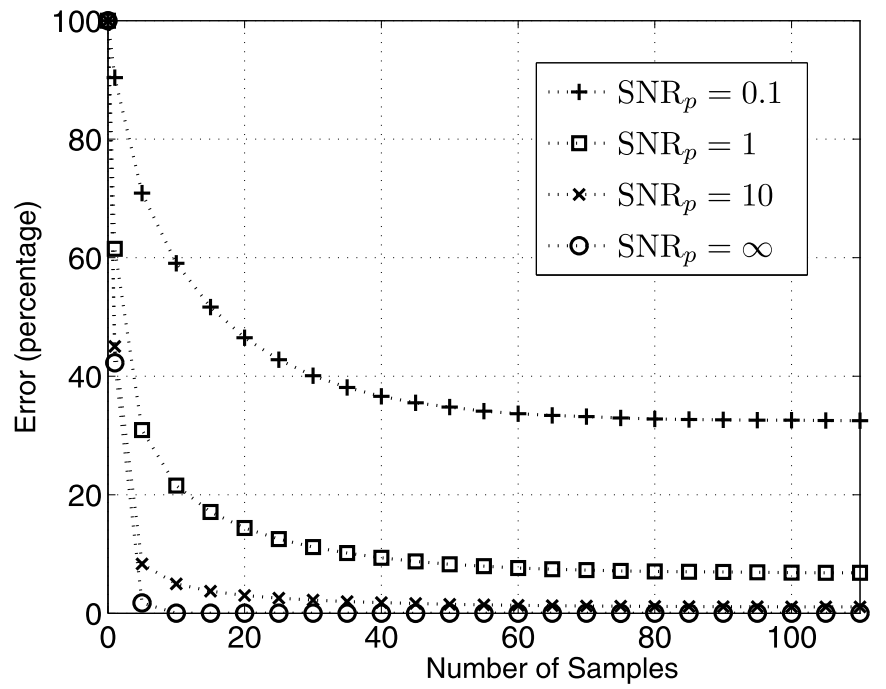

Fig. 3. Error vs number of samples, $\beta=1$ (varying $\mathrm{SNR}_{p}$ ).

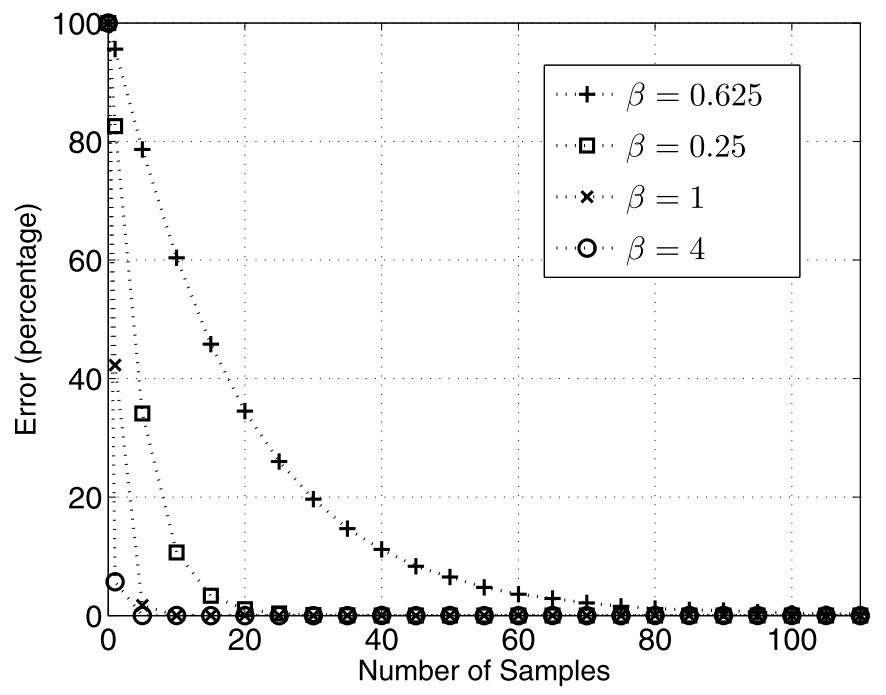

Fig. 4. Error vs number of samples, $\operatorname{SNR}_{p}=\infty$ (varying $\beta$ ).

\subsubsection{Variable Effective DOF}

We now investigate the effect of the DOF of the unknown field on the trade-off between $M_{b}$ and $\boldsymbol{\varepsilon}\left(M_{b}\right)$. Here $\beta$ is varied over $\beta=[1 / 16,1 / 4,1,4]$ and two different values of $\operatorname{SNR}_{p}=[0.1, \infty]$ are considered. Figs. 4 and 5 show the results for $\operatorname{SNR}_{p}=\infty$ and $\mathrm{SNR}_{p}=0.1$, respectively. Both of the plots show that for lower values of $\beta$ (corresponding to higher DOF), it is more difficult to achieve low values of error within a given number of samples. But as $\beta$ increases, the total uncertainty in the field decreases, and it becomes a lot easier to achieve lower values of error.

In Fig. 4, we observe that for all values of $\beta$, effectively zero error is obtained as $M_{b}$ is increased; the field can be represented with effectively 0 error with a finite number of samples. This is not surprising, since the effective DOFs of the signal sources under consideration are finite.

Comparing the performances in Figs. 4 and 5 for low and high values of the cost budget, we see that the effect of DOF is more pronounced for different $\mathrm{SNR}_{p}$ values for different regions of $M_{b}$ : for low $M_{b}$ values, the effect of DOF is more strong in the high $\mathrm{SNR}_{p}$ case; for high $M_{b}$ values, the effect of DOF is more strong in the low $\mathrm{SNR}_{p}$ case. For low $M_{b}$ values, for the high $\mathrm{SNR}_{p}$ case there is a drastic performance difference between different values

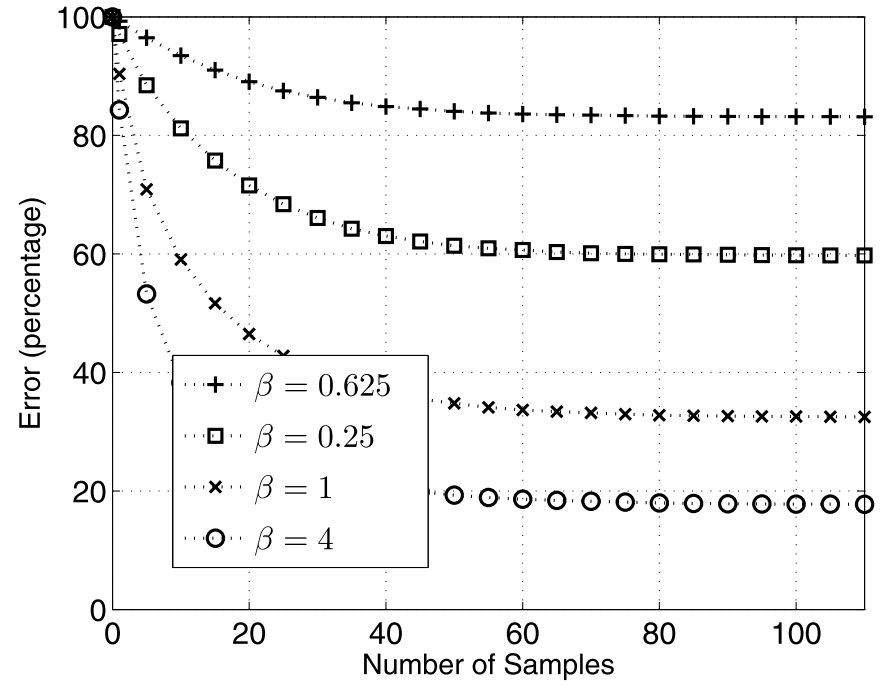

Fig. 5. Error vs number of samples, $\operatorname{SNR}_{p}=0.1$ (varying $\beta$ ).

of DOF; for the lower DOF values it is possible to obtain very low values of error $(\approx 0)$, a far better performance compared to the higher DOF case. As $M_{b}$ increases, the difference in performance for different values of DOF decreases, and effectively zero error is obtained for all values of DOF. For high $M_{b}$ values, the effect of DOF is more pronounced in the low $\mathrm{SNR}_{p}$ case: the error curves for fields with different DOFs saturate at different values. When the noise level is high, it is not possible to wash out the effect of system noise by taking more samples if the fields have high DOF, hence the curves saturate at relatively high error values. On the other hand, the effect of noise can be canceled out if the field has relatively low DOF, hence these curves saturate at relatively low values.

\subsection{Trade-offs and the optimum sampling interval}

In this section we will investigate the relationship between the optimum sampling interval $\Delta$ and the problem parameters $M_{b}, \beta$, $\mathrm{SNR}_{p}$.

In general, the optimum policy under a given number of samples can be informally interpreted in the light of two driving forces. The first one is to collect as many effectively uncorrelated samples as possible, so that every sample we have will provide as much new information as possible about the field. The other one is to avoid samples with low variances, since a sample with a low variance is worse than a sample that has higher variance and has the same correlation coefficient with the field values at other points (so that the amount of uncertainty reduction for the other field values due to observation of this sample will be the same). We note that for a GSM source the function that determines the correlation of a field value at a particular point with the field values at other points is the same for a field value at any given location (given by $v\left(x_{1}, x_{2}\right)$ ), and it is a decreasing function of the distance between the points. Hence when we take a sample at a particular point, we also obtain some information about the field values around that point, but not so much about the field values that are far away. Due to the GSM source structure, low variance samples have relatively low variance neighbors, and hence the decrease in the uncertainty due to observation of field values at these points will be relatively low. This further encourages us to avoid samples with low variances.

\subsubsection{Optimum sampling interval}

Here we investigate the dependence of the optimum sampling interval on $\beta, \operatorname{SNR}_{p}$ and $M_{b}$. 


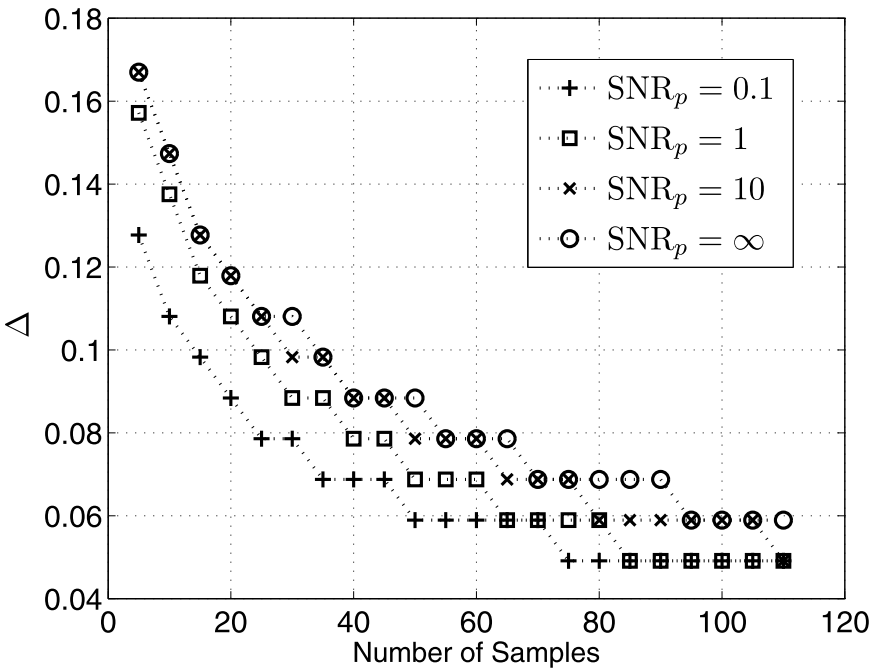

Fig. 6. Optimum sampling interval vs number of samples, $\beta=1 / 16$ (varying $\mathrm{SNR}_{p}$ ).

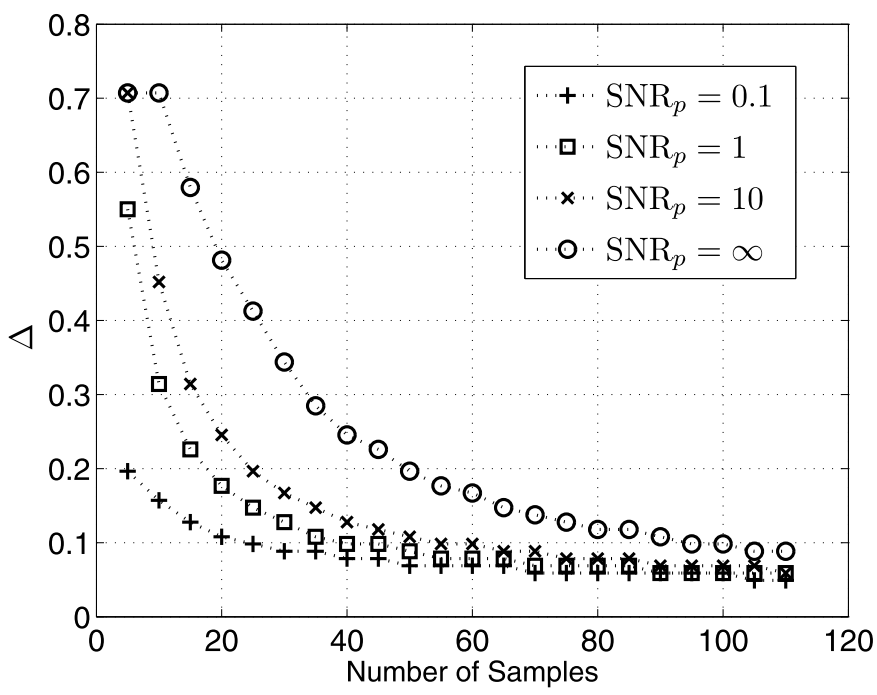

Fig. 7. Optimum sampling interval vs number of samples, $\beta=1$ (varying $\mathrm{SNR}_{p}$ ).

Figs. 6 and 7 give the optimum sampling intervals versus number of samples for $\beta=1 / 16$ and $\beta=1$, respectively. We observe that in general the optimum sampling interval decreases with increasing number of samples. When the number of samples one is allowed is low, one tries to obtain as much independent information as possible by choosing the samples apart. As $M_{b}$ increases and we are allowed to use more samples, one can afford to choose the samples closer so that field values that were considered to give enough information about each other in the former case can be also observed and lower values of error can be obtained.

For a given $\beta$ and $M_{b}$, the sampling interval increases with increasing $\mathrm{SNR}_{p}$. As $\mathrm{SNR}_{p}$ increases, observing the field at a particular point allows one to estimate the value of the field at this point and its neighbors better. Therefore, to ensure that each sample provides new information, one should increase the sampling interval.

Comparing Figs. 6 and 7, we observe that the optimum sampling intervals are smaller for the high DOF case (Fig. 6). As DOF increases, that is, the number of uncorrelated random variables required to effectively represent the field increases, and also given the GSM correlation structure, the field value at each point becomes less correlated with its neighboring points. Hence the reduction in the uncertainty of the field values at the neighbors of

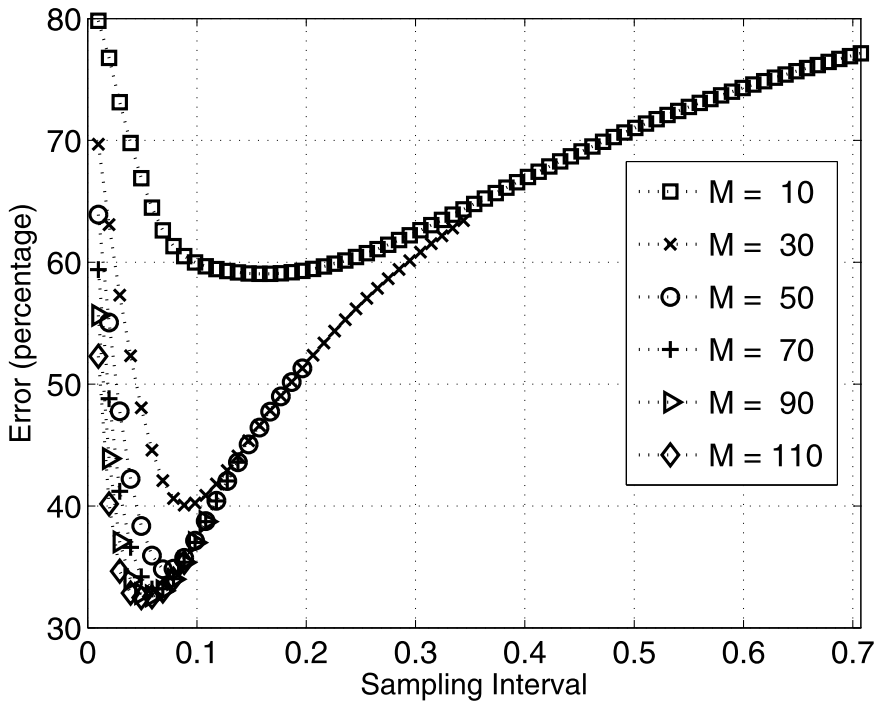

Fig. 8. Error vs sampling interval, $\beta=1, \mathrm{SNR}_{p}=0.1$ (varying number of samples).

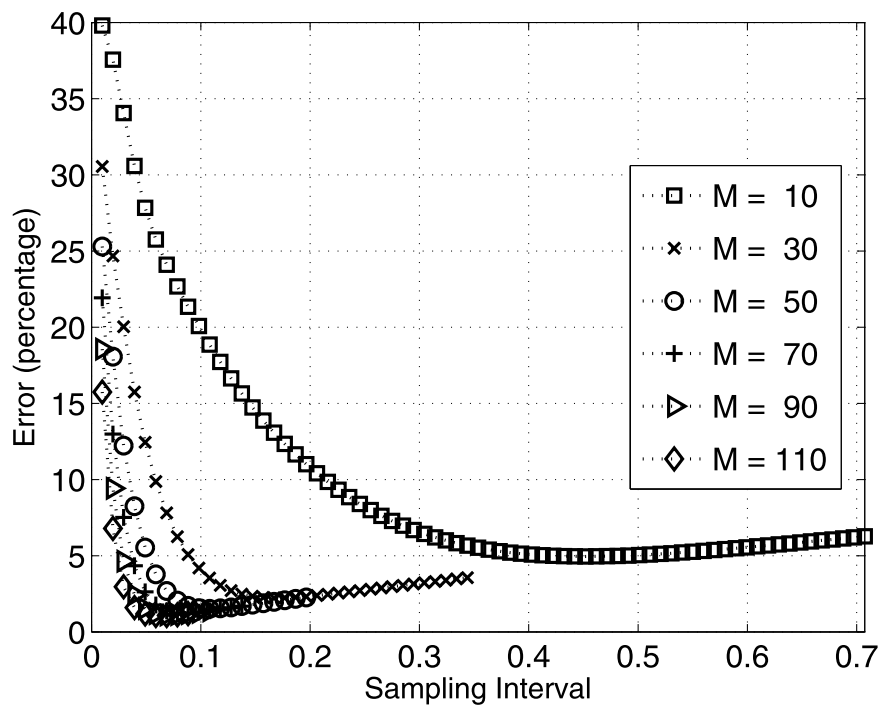

Fig. 9. Error vs sampling interval, $\beta=1, \mathrm{SNR}_{p}=10$ (varying number of samples).

a given point due to the observation of the field at a this point is smaller. This, together with the fact that the variances of field values decrease as the samples are placed further away from $x=0$ point, encourages us to take samples more closely, so that all the effectively uncorrelated samples with high variances can be collected.

\subsubsection{Sensitivity of performance to the sampling interval}

In this section we investigate the sensitivity of the performance to the sampling interval. For this purpose we look at the error versus sampling interval curves and observe how much the error deviates from its optimum value as the sampling interval deviates from the optimum sampling interval.

Figs. 8, 9, 10 and 11 present the error versus sampling interval curves for $\beta=1, \mathrm{SNR}_{p}=0.1$, and $\beta=1, \mathrm{SNR}_{p}=10$, and $\beta=1 / 16$, $\mathrm{SNR}_{p}=10$, and $\beta=1 / 16, \mathrm{SNR}_{p}=0.1$, respectively. We note that in all figures, as $M$ increases, data for fewer numbers of sampling points are plotted. This is due to the fact that we only allow the samples to be taken in the bounded domain $D$, and as $M$ increases, larger sampling intervals become impermissible. 


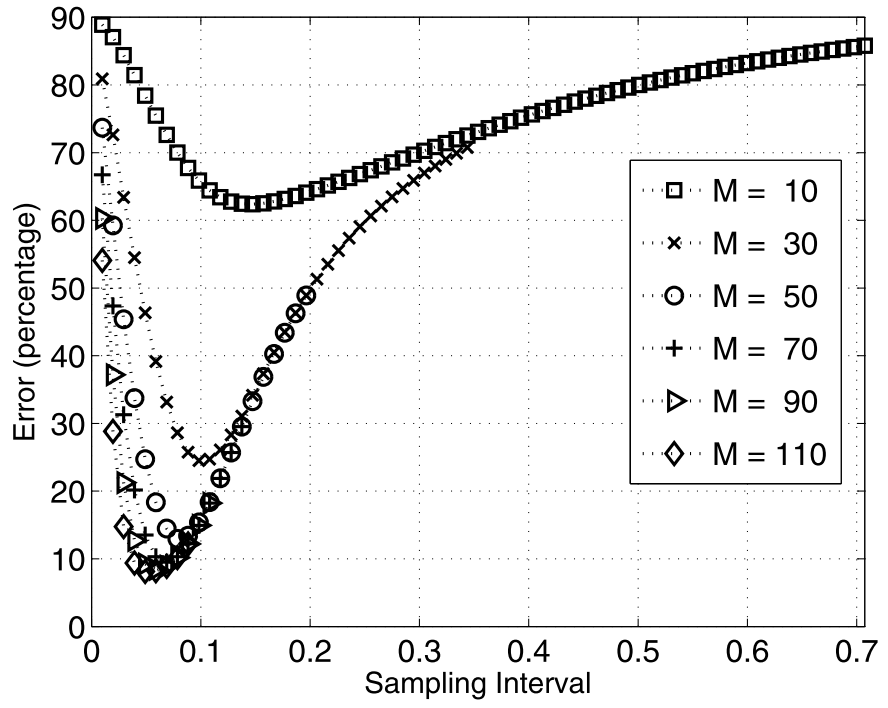

Fig. 10. Error vs sampling interval, $\beta=1 / 16, \mathrm{SNR}_{p}=10$ (varying number of samples).

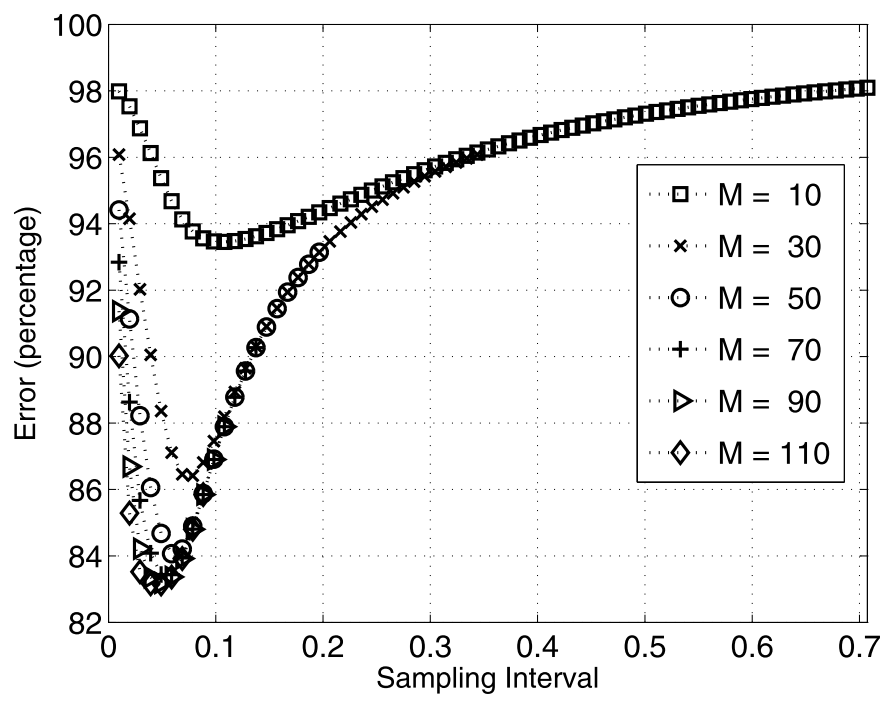

Fig. 11. Error vs sampling interval, $\beta=1 / 16, \mathrm{SNR}_{p}=0.1$ (varying number of samples).

We observe that in all of these figures, for a given $M$ the error first decreases as we increase the sampling interval, and after reaching the optimum sampling interval it starts to increase again. This behavior may be interpreted in view of the following observation: We expect that the optimum policy will be the one that takes as many uncorrelated samples with high variances as possible. If we take the samples too close, we acquire random variables close to each other whose correlation will be relatively strong due to the nature of the GSM model. Hence the error will be relatively high, since the samples are spent on obtaining redundant information. On the other hand, if we take the samples far apart from each other, we may be missing some of the random variables that contain effectively uncorrelated information with the samples we take. Moreover, we may waste our sample budget on random variables that have relatively low variance (the ones that are outside the main lobe of the Gaussian intensity function). Hence the error may again be relatively high.

While commenting on the sensitivity, we focus on the differences in absolute error in different scenarios. We observe that, for a given $\beta$ and $\mathrm{SNR}_{p}$, as $M$ increases, the achievable error values become more sensitive to the sampling interval. For instance, in Fig. 8 for $M=10$, any sampling interval in the range [ 0.10 .25 ] provides approximately the same error $(\approx 60 \%)$; whereas for $M=70$, a similar range of sampling intervals around the optimum sampling interval (such as $[0.020 .15]$ ) produce error values in the range of $\approx 35-50 \%$. When we are allowed a small number of samples, taking samples with a high enough sampling interval can easily provide effectively uncorrelated samples; avoiding samples with low variances is not a serious issue that requires sensitive design, choosing the sampling interval smaller than a given value is enough. Hence any sampling interval between these lower and higher bounds produces effectively the same error level with the optimum interval. On the other hand, when a larger number of samples are allowed, one has to design the locations of the samples more carefully to find the best trade-off between collecting relatively uncorrelated samples and avoiding samples with low variances.

We observe that when DOF is low, the error may be considered to be more sensitive to the sampling interval for low $\mathrm{SNR}_{p}$ values. For instance, for $\beta=1, \mathrm{SNR}_{p}=10$, and $M=10$, any sampling interval in the range $\left[\begin{array}{ll}0.3 & 0.6\end{array}\right]$ provide approximately the same error with the optimal sampling strategy $(\approx 5 \%)$. On the other hand, for $\beta=1, \mathrm{SNR}_{p}=0.1$, in order to have approximately the same error with the optimal strategy $(\approx 60 \%)$, only sampling intervals in the range $\left[\begin{array}{ll}0.1 & 0.25\end{array}\right]$ are allowed. We note that the length of [0.1 0.25$]$ is half of the length of [0.3 0.6]. On the other hand, when DOF is high, the error is more sensitive to the sampling interval for high $\mathrm{SNR}_{p}$ values. We remind that in these comparisons we consider the variation in absolute error for different scenarios. For instance, for $\beta=1 / 16, \operatorname{SNR}_{p}=0.1$, and $M_{b}=10$, in order to obtain an error that is not worser than the error obtained with the optimal strategy by more than $5 \%$ percent $(\approx 93-98 \%$ ), it is sufficient to use any sampling interval in the range of [0.01 0.7]. On the other hand, for $\beta=1 / 16, \mathrm{SNR}_{p}=10$, in order to obtain an error that is not worser than the error obtained with the optimal strategy by more than $5 \%$ percent, $(\approx 60-65 \%)$, it is necessary to use a sampling interval in the range of [0.1 0.2$]$, a significantly smaller range.

Similar comparisons can be made for the other cases as well: When $\mathrm{SNR}_{p}$ is high/low, the sensitivity of the error to the sampling interval increases with increasing/decreasing DOF. All of these results concerning the sensitivity can be interpreted in the light of the following observation: In general, we observe that the error becomes more sensitive to our choice of sampling interval when the effect of different problem parameters on the optimum sampling interval conflict: One of the problem parameters requires us to take the samples closer to each other, while the other requires us to take them farther apart. For instance, low DOF requires us to take the samples apart whereas low $\mathrm{SNR}_{p}$ requires us to take the samples closer. Hence for low DOF, as $\mathrm{SNR}_{p}$ decreases, the error becomes more sensitive to the sampling interval. Taking a closer look, we observe that when DOF is low, the field values are highly correlated with each other, and for high values of $\mathrm{SNR}_{p}$ the field values to be observed contain low levels of noise. Hence the samples carry essentially the same information, making the choice of the sampling interval relatively unimportant. As $\mathrm{SNR}_{p}$ decreases, a compromise between the two conflicting forces is required, making this choice more important: taking samples close enough so that the noise is effectively washed out, and taking samples sufficiently apart from each other so that each sample brings new information.

\section{Conclusions}

We have considered the representation of a finite-energy nonstationary random field with a finite number of samples. By considering a parametric non-stationary field model, namely the 
Gaussian-Schell model, we obtained the trade-offs between the number of samples and the representation error, for varying noise levels and for sources with varying degrees of freedom (DOF). We have discussed the optimum sampling intervals, and their dependence on the problem parameters. We have observed that increases in either of (i) the number of allowed samples, (ii) DOF, or (iii) the noise level, results in a decrease in the optimum sampling interval. We have also investigated the sensitivity of the error to the chosen sampling interval. We have observed that the error is more sensitive to sampling interval when (i) the number of allowed samples is high, (ii) DOF is high and the noise level is low, or (iii) DOF is low and the noise level is high.

\section{Acknowledgments}

A. Özçelikkale was supported by TÜBITAK Doctoral Scholarship. H.M. Ozaktas acknowledges partial support of the Turkish Academy of Sciences.

\section{References}

[1] A. Balakrishnan, A note on the sampling principle for continuous signals, IRE Trans. Inf. Theory 3 (2) (1957) 143-146.

[2] S.P. Lloyd, A sampling theorem for stationary (wide sense) stochastic processes, Trans. Am. Math. Soc. 92 (1) (1959) 1-12.

[3] F.J. Beutler, Sampling theorems and bases in a Hilbert space, Inf. Control $4(2-3)$ (1961) 97-117.

[4] F.J. Beutler, Error-free recovery of signals from irregularly spaced samples, SIAM Rev. 8 (3) (1966) 328-335.

[5] M.I. Kadec, The exact value of the Paley-Wiener constant, Dokl. Akad. Nauk SSSR 155 (1964) 1253-1254

[6] R.M. Young, An Introduction to Nonharmonic Fourier Series, Academic Press, New York, 1980

[7] P. Babu, P. Stoica, Spectral analysis of nonuniformly sampled data - a review, Digit. Signal Process. 20 (2) (2010) 359-378.

[8] E. Masry, On the truncation error of the sampling expansion for stationary bandlimited processes, IEEE Trans. Signal Process. 42 (10) (1994) 2851-2853.

[9] Z. Song, W. Sun, X. Zhou, Z. Hou, An average sampling theorem for bandlimited stochastic processes, IEEE Trans. Inf. Theory 53 (12) (2007) 4798-4800.

[10] W.A. Gardner, A sampling theorem for nonstationary random processes, IEEE Trans. Inf. Theory 18 (6) (1972) 808-809.

[11] F. Garcia, I. Lourtie, J. Buescu, $L_{2}(R)$ nonstationary processes and the sampling theorem, IEEE Signal Process. Lett. 8 (4) (2001) 117-119.

[12] A. Napolitano, Sampling theorems for Doppler-stretched wide-band signals, Signal Process. 90 (7) (2010) 2276-2287.

[13] A. Napolitano, Sampling of spectrally correlated processes, IEEE Trans. Signal Process. 59 (2) (2011) 525-539.

[14] W.A. Gardner, A. Napolitano, L. Paura, Cyclostationarity: Half a century of research, Signal Process. 86 (4) (2006) 639-697.

[15] T. Michaeli, Y. Eldar, High-rate interpolation of random signals from nonideal samples, IEEE Trans. Signal Process. 57 (3) (2009) 977-992.

[16] A. Özçelikkale, H.M. Ozaktas, E. Arıkan, Signal recovery with cost constrained measurements, IEEE Trans. Signal Process. 58 (7) (2010) 3607-3617.

[17] P. Flandrin, A. Napolitano, H.M. Ozaktas, D.J. Thomson, Recent advances in theory and methods for nonstationary signal analysis, Special Issue of EURASIP j. Adv. Signal Process. (2011).

[18] H.L. Van Trees, Detection, Estimation and Modulation Theory, Part I, Wiley, New York, 2001
[19] A. Starikov, E. Wolf, Coherent-mode representation of Gaussian-Schell model sources and of their radiation fields, J. Opt. Soc. Am. A 72 (7) (1982) 923-928.

[20] A.T. Friberg, R.J. Sudol, The spatial coherence properties of Gaussian-Schell model beams, Opt. Act. 30 (8) (1983) 1075-1097.

[21] A.T. Friberg, J. Turunen, Imaging of Gaussian-Schell model sources, J. Opt. Soc. Am. A 5 (5) (1988) 713-720.

[22] F. Gori, Collett-Wolf sources and multimode lasers, Opt. Commun. 34 (3) (1980) 301-305.

[23] H. Yoshimura, T. Iwai, Properties of the Gaussian-Schell-model source field in a fractional Fourier plane, Opt. Act. 14 (12) (1997) 3388-3393.

[24] Q. Lin, Y. Cai, Fractional Fourier transform for partially coherent GaussianSchell model beams, Opt. Lett. 27 (19) (2002) 1672-1674.

[25] T. Shirai, A. Dogariu, E. Wolf, Directionality of Gaussian-Schell-model beams propagating in atmospheric turbulence, Opt. Lett. 28 (8) (2003) 610-612.

[26] Y. Dan, S. Zeng, B. Hao, B. Zhang, Range of turbulence-independent propagation and Rayleigh range of partially coherent beams in atmospheric turbulence, J. Opt. Soc. Am. A 27 (3) (2010) 426-434.

[27] A. Papoulis, Probability, Random Variables, and Stochastic Processes, 3rd ed., McGraw-Hill, 1991.

[28] W. Lukozs, Optical systems with resolving powers exceeding the classical limit, J. Opt. Soc. Am. 56 (11) (1966) 1463-1472.

[29] G. Toraldo di Francia, Degrees of freedom of an image, J. Opt. Soc. Am. 59 (7) (1969) 799-804.

[30] F. Gori, in: R.J. Marks II (Ed.), Advanced Topics in Shannon Sampling and Interpolation Theory, Springer-Verlag, New York, 1993, pp. 37-83, Ch. 2

[31] A. Poon, R. Brodersen, D. Tse, Degrees of freedom in multiple-antenna channels: a signal space approach, IEEE Trans. Inf. Theory 51 (2) (2005) 523-536.

[32] R. Kennedy, P. Sadeghi, T. Abhayapala, H. Jones, Intrinsic limits of dimensionality and richness in random multipath fields, IEEE Trans. Signal Process. 55 (6) (2007) 2542-2556.

[33] R.A. Horn, C.R. Johnson, Matrix Analysis, Cambridge University Press, 1990.

[34] J. Buescu, Positive integral operators in unbounded domains, J. Math. Anal. Appl. 296 (1) (2004) 244-255.

Ayça Özçelikkale received the B.S. degree from Middle East Technical University, Ankara, in 2004, and the M.S. degree and the Ph.D. degree from Bilkent University, Ankara, in 2006, and in 2012, respectively. Her current research interests are in the area of signal processing and communications.

Haldun M. Ozaktas received the B.S. degree from Middle East Technical University, Ankara, in 1987 and the Ph.D. degree from Stanford University, Stanford, California, in 1991. He joined Bilkent University, Ankara, in 1991, where he is presently a Professor of electrical engineering. In 1992, he was at the University of Erlangen-Nuremberg, Bavaria as an Alexander von Humboldt Foundation Postdoctoral Fellow. During summer 1994, he worked as a Consultant at Bell Laboratories, Holmdel, New Jersey. He is the author of over 100 refereed journal articles, over 12 book chapters, and over 110 conference presentations and papers, over 45 of which have been invited. He is also author of the book The Fractional Fourier Transform (Wiley, 2001) and edited the book Three-Dimensional Television (Springer, 2008). His academic interests include signal and image processing, optical information processing, and optoelectronic and optically interconnected computing systems. Dr. Ozaktas has a total of over 4800 citations to his work recorded in the Science Citation Index (ISI). $\mathrm{He}$ is the recipient of the 1998 ICO International Prize in Optics and one of the youngest recipients ever of the Scientific and Technical Research Council of Turkey (TUBITAK) Science Award (1999), among other awards and prizes. He is also one of the youngest-elected members of the Turkish Academy of Sciences and a Fellow of the OSA and the SPIE. 